\title{
SIBR: A Routing Protocol for Delay Tolerant Networks
}

\author{
Yuan Chen \\ Dept. of Computer Science and Technology \\ East China Normal University \\ Shanghai, China \\ E-mail: chenyuancs@gmail.com
}

\author{
Neng Wang \\ Dept. of Computer Science and Technology \\ East China Normal University \\ Shanghai, China \\ E-mail: nwang@cs.ecnu.edu.cn
}

\begin{abstract}
Delay tolerant networks (DTNs) may lack continuous network connectivity. Routing in DTNs is thus challenging since it must handle network partitioning, long delays, and dynamic topology in such networks. Most routing protocols for delay tolerant networks resort to the sufficient state information, including trajectory and contact information, to ensure routing efficiency. In this paper, we propose an interconnection social metric of each node in the network to perform the routing process. We propose a new protocol based on social community and social interconnection of the nodes to maximize data delivery. Results show that the proposed protocol achieves higher delivery ratio and less average delay compared to PROPHET and SimBet routing protocols.
\end{abstract}

Keywords-Delay tolerant networks; Routing; Social community.

\section{INTRODUCTION}

DELAY or disruption tolerant networks (DTNs) [1]-[3] have recently drawn much attention from networking researchers due to the wide applications of these networks in challenging environments, such as space communications, military operations, and mobile sensor networks. Intermittent connectivity in DTNs results in the lack of instantaneous end-to-end paths, large transmission delay and unstable network topology.

Traditional routing protocols for wired and wireless networks fail to work in the DTN environment because they assume the existence of continuous end-to-end connections between sources and destinations. Routing protocols developed for DTN should be adapted to this challenging environment by sending multiple copies of data packets to increase the probability that one of the copies reaches the destination. Nodes receiving the packet copies store them until they meet other nodes or meet their destinations.

Our objective is to develop a routing protocol that spreads a small number of packet copies to reduce network overhead, while guiding the packet copies using local information to reach the destination. To achieve that goal, we exploit the social community characteristic of DTN nodes. We consider two nodes to belong to the same social community if they contact each other frequently compared to their contacts with other nodes. We claim that our work is the first to propose an interconnection social metric, which sprays messages by considering nodes' history contacts of community. We consider some nodes have higher social value if they contact more different nodes from more different communities. If we select these nodes as relay nodes, the coverage of messages will be wider and forwarding performance will be improved. More explanation is provided in Section 3.

The major contributions of our work are as follows:

1 . We are the first to propose an interconnection social metric.

2. A new heuristic multiple-copy routing protocol, Social Interconnection Based Routing (SIBR), is proposed. The protocol exploits social Interconnection among network community to increase the packet delivery probability, without flooding the network with many redundant copies.

3. We evaluate the proposed scheme using ONE [4], a simulation tool. The simulation results show the competitive performance of SIBR in DTNs.

The rest of the paper is organized as follows. Section 2 shows the related work. Section 3 explains the new SIBR routing protocol. Section 4 focuses on the simulation and evaluation. We summarize the work in Section 5.

\section{RELATED WORKS}

For routing and forwarding in DTNs and mobile ad hoc networks, there is much existing literature. Many approaches calculate the probability of delivery to the destination node, where the metrics are derived from the history of node contacts, spatial information and so forth. The pattern-based Mobyspace Routing by Leguay et al. [5], location-based routing by Lebrun et al. [6], context-based forwarding by Musolesi et al. [7] and PROPHET Routing [8] fall into this category.

PROPHET Routing is probability-based, using past encounters to predict the probability of meeting a node again. Nodes that are encountered frequently have an increased probability whereas older contacts are degraded over time. Additionally, the transitive nature of encounters is exploited where nodes exchange encounter probabilities and the probability of indirectly encountering the destination node is evaluated.

Recent attempts to uncover a hidden stable network structure in DTNs such as social networks have been emerged. SimBet Routing [9] studies the "small-world" phenomenon of human society and uses ego-centric centrality and its social similarity to guide data forwarding. Messages are forwarded towards the node with higher centrality. LABEL forwarding [10] uses affiliation information to help forwarding in PSNs based on the simple intuition that people belonging to the same community are likely to meet frequently, and thus act as suitable forwarders 
for messages destined for members of the same community. Using social connections has been addressed in previous papers as in [8], [9], [10], [11], [12], and [13]. All of the previous work focused on using inclusive social metrics, which predicts the path from source to destination by including nodes with strong social connections.

\section{DESCRIPTION OF SIBR}

\section{A. Protocol Description}

The proposed protocol aims to utilize the community and interconnection property of nodes. An example of such community and interconnection would be the taxi companies in a big city. Taxis that belong to the same companies contact each other more frequently, because they either meet in the city roads or in the company garage during their break times. Therefore, taxis that belong to the same company are considered to have strong social connection among each other. If there is a source taxi in one company want to send a message to another destination taxi in another destination company, it can copy and send the message to any taxis in any other companies it encounters. And then these taxis can do the same procedure. When the time goes by, the message will spread into wider and wider space and reach some taxi in destination company. Finally the message will reach the destination taxi.

We consider nodes, frequently meeting each other, to belong to the same social community where they are expected to meet each other again frequently. They are also expected to have the same social relation with other nodes. In that sense, each node may consider itself a representative of the community to distribute its packets to other community. From our protocol perspective, it is useless to keep several copies of the same packet inside one social community. Therefore, a node that has a packet destined to other nodes outside its community tends to forward the packet copies to other communities. At the same time, there exist some nodes that often encounter other nodes from other communities. We select these nodes as relay nodes which transfer messages to other nodes from other communities. So the coverage of messages will be wider and forwarding performance will be improved.

\section{B. Protocol Design}

We assume that nodes have known which community they belong to. Every node has properties: its id, its community id, Packet Summary Vector, Encountered Communities Summary Vector and Delivered Packets Summary Vector.

Details of the protocol are explained in the following:

1 Each packet generated is assigned a unique packet ID. The list of all the packet IDs in a node's buffer is called the Packet Summary Vector.

2 Each node has The Encountered Communities Summary Vector which has properties: community ID and encountered times. It records communities node has reached and encountered times of each community.
3 The Delivered Packets Summary Vector has properties: Packet ID, community ID and symbol which indicates if the certain packet has been delivered to the certain community.

4 If two contacting nodes come from different community, they check if the other's community id exists in its Encountered Communities Summary Vector. If not, it means that they meet the first time, and then following steps will be taken:

4.1 They add the other's community id to its Encountered Communities Summary Vector each other, which means that they have reached the other's community;

4.2 They duplicate and deliver packets to the newly encountered node each other;

4.3 They update the Delivered Packets Summary Vector each other. If the item [packet id, community id, *] exists, then update it to [packet id, community id, 1], otherwise add [packet id, community id, 1] to the Delivered Packets Summary Vector. It means they have delivered packets to the other's community.

5 If two contacting nodes come from different community and they have ever met before, following steps will be taken:

5.1 Merge their Delivered Packets Summary Vector. If [packet id, community id, 1] exists in one's Delivered Packets Summary Vector, then update [packet id, community id, *] in the other's Delivered Packets Summary Vector to [packet id, community id, 1]. It means that one node has delivered packets to some communities and the other node needn't deliver the same packets to the same communities.

5.2 According to its Delivered Packets Summary Vector, node can get all the packets that have been delivered to the other node's community. And then with its Packet Summary Vector, node can infer what packets haven't been delivered to the other node's community. So it duplicate and send undelivered packets to the other node and update its Delivered Packets Summary Vector.

6. If two contacting nodes come from the same community, following steps will be taken:

6.1 If one node's Encountered Communities Summary Vector contains the other's Encountered Communities Summary Vector, which means it have reached all communities $\mathrm{n}$ have reached, the other must deliver all the packets to it.

6.2 If their Encountered Communities Summary Vector are different, which means they have been to different communities, they do the same procedure as protocol detail 5 .

Fig. 1 shows the process performed by contacting node in details. 


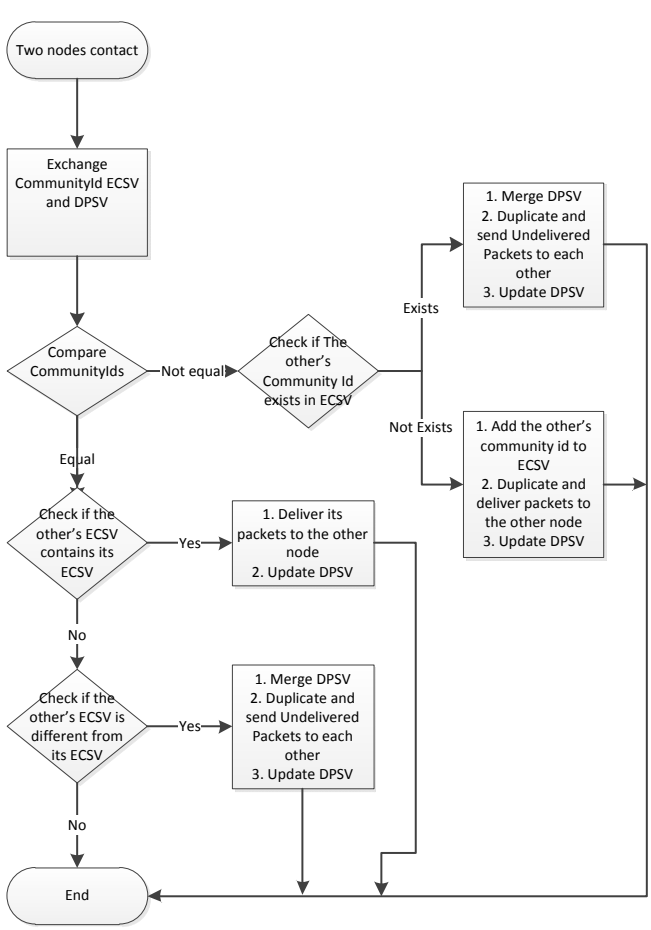

Figure 1. The process performed by contacting node

\section{PERFORMANCE EVALUATION}

In this section we describe the simulations used to evaluate SIBR Routing and compare its cost and performance to PROPHET Routing and SimBet Routing. To perform our evaluation, we use the Opportunistic Network Environment simulator (ONE), which is a simulation environment designed specifically for delay tolerant networks. We consider following two evaluation metrics:

1 Average message delivery ratio: measured as the relation of the number of unique delivered messages to the number of messages sent;

2 Average end-to-end message latency: measured as the time between message creation and delivery.

\section{A. Mobility Models}

The vehicular-based map-driven model, which is part of the ONE simulator, limits node movement to actual streets found on an imported map, an approximate $4.5 \mathrm{~km} \times 3.4 \mathrm{~km}$ section of downtown Helsinki, Finland. The model contains 120 nodes, which is divided into 3 communities and every community contains 40 nodes. Each community of nodes is randomly distributed in a map of three adjacent sub-regions. The average moving speeds of each community of nodes are $1.34 \mathrm{~m} / \mathrm{s}, 4 \mathrm{~m} / \mathrm{s}$ and $8.94 \mathrm{~m} / \mathrm{s}$, which are the average speed of walking, jogging and biking. Nodes' downtime are $0 \sim 120$ s, communication range is $10 \mathrm{~m}$, and transmission speed is 2 Mbps. The nodes cache size changes from $100 \mathrm{~kb}$ to $500 \mathrm{~kb}$. The scene randomly selected node pair to transmit and receive messages and each message is $1 \mathrm{~kb}$. Message generation time interval respectively is $10 \sim 20 \mathrm{~s}$. The simulation time is 4.5 hour, and Warm-up time is $1000 \mathrm{~s}$ before start the simulation, during which no messages are produced.

\section{B. Performance Comparison under Different Cache}

Figure 2 and 3 shows a comparison of the respective performance of the three algorithms when cache size changes while the TTL of message is $150(\mathrm{~min})$. Figure 2 shows that the delivery rate of the various algorithms increases with cache size increasing. This is because each node can accommodate more number of messages and the number of copies of each packet to be passed to the destination node increases, the final delivery ratio improves. Because SIBR routing considers it useless to keep several copies of the same packet inside one social community, so it produce less packets and its performance is better than the others. Figure 3 illustrates the increase in the number of copies of the packets means that there are more nodes to pass a packet, so the average latency reduces.

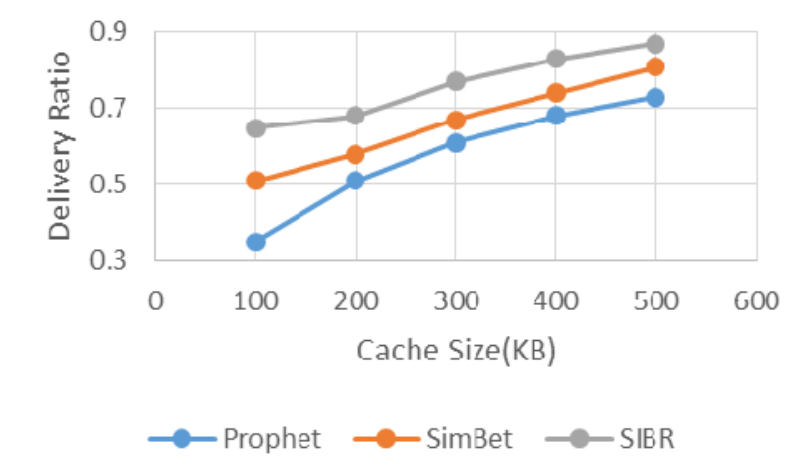

Figure 2. Delivery Ratio Comparison under Different Cache

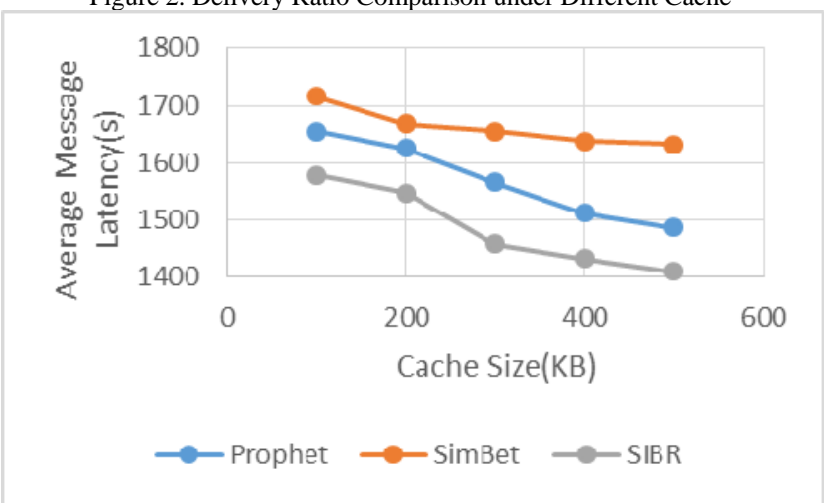

Figure 3. Average Message Latency Comparison under Different Cache

\section{Performance Comparison under Different TTL}

Figure 4 and 5 shows a comparison of the respective performance of the three algorithms when the TTL of message changes while cache size is $300 \mathrm{~kb}$. Figure 4 shows that the delivery rate of the various algorithms increases with TTL increasing. This is because messages can exist longer time when TTL increases, more messages can reach destination node. Figure 5 illustrates while the TTL increases, the average end-to-end message latency also increases. Because SIBR routing can make message spread wider space sooner, its average message latency is less. 


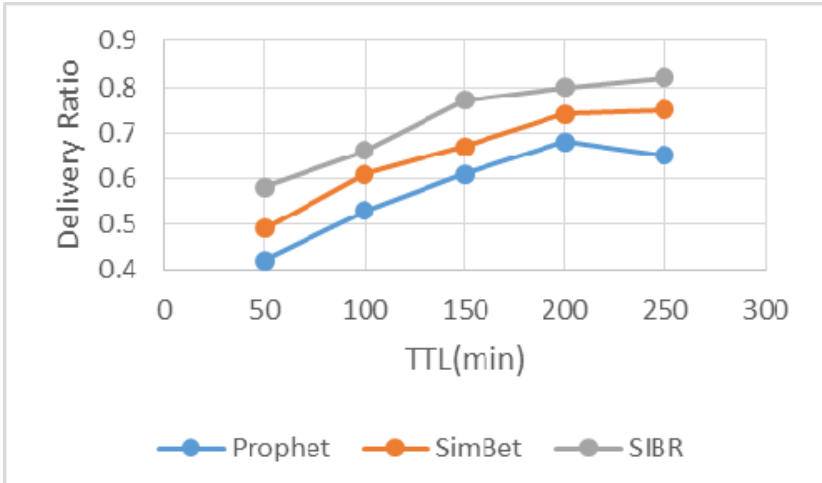

Figure 4. Delivery Ratio Comparison under Different TTL

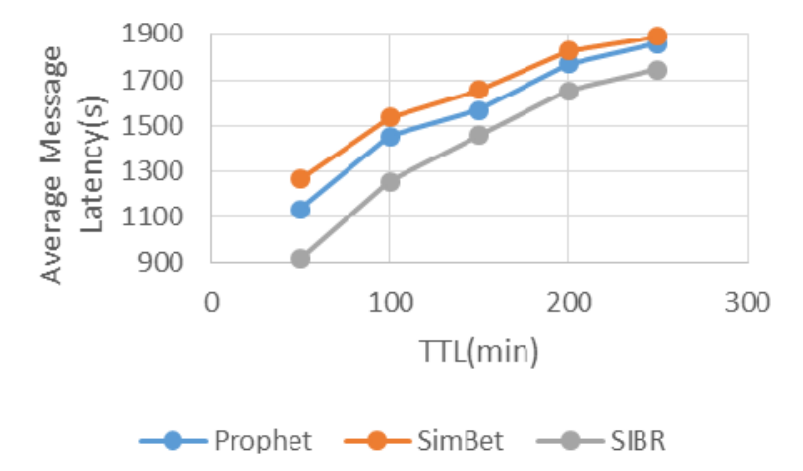

Figure 5. Average Message Latency Comparison under Different TTL

\section{CONCLUSION}

Delay Tolerant Networks (DTN), lack end-to-end connections between data sources and destinations. This requires the intermediate nodes to store data packets for long periods of time which violates one of the basic assumptions of traditional routing protocols and triggers the development of new ones. We have shown that it is possible to uncover important characteristic properties of social network. We have demonstrated that community and interconnection social metrics can be effectively used in forwarding decisions. Our SIBR algorithm is designed for a delay tolerant network environment and we have shown that it has higher delivery ratio and less average delay compared to PROPHET and SimBet routing protocols.

\section{REFERENCES}

[1] Z. Zhang, "Routing in intermittently connected mobile ad hoc networks and delay tolerant networks: overview and challenges," IEEE Commun. Surveys Tutorials, vol. 8, no. 1, pp. 24-37, 2006.

[2] Y. Wang, H. Dang, and H. Wu, “A survey on analytic studies of delay-tolerant mobile sensor networks” Wirel. Commun. Mob. Comput., vol. 7, no. 10, pp. 1197-1208, 2007.

[3] Y. Zhu, B. Xu, X. Shi, and Y. Wang, “A survey of social-based routing in delay tolerant networks: positive and negative social effects,” IEEE Communications Surveys and Tutorials, pp (99), 1-15, 2012.

[4] ONE simulator. http://www.netlab.tkk.fi/tutkimus/dtn/theone/.

[5] J. Leguay, T. Friedman, and V. Conan, "Evaluating mobility pattern space routing for DTNs,” in Proc. INFOCOM, 2006.

[6] J. Lebrun, C.-N. Chuah, D. Ghosal, and M. Zhang, "Knowledgebased opportunistic forwarding in vehicular wireless ad hoc networks,” IEEE VTC, vol. 4, pp. 2289-2293, 2005.

[7] M. Musolesi, S. Hailes, et al., "Adaptive routing for intermittently connected mobile ad hoc networks,” in Proc. WOWMOM, 2005.

[8] A.Lindgren, A.Doria, and O.Schelen, "Probabilistic routing in intermittently connected networks,” in Proc. SAPIR, 2004.

[9] E. Daly and M. Haahr, "Social network analysis for routing in disconnected delay-tolerant manets," in Proceedings of ACM MobiHoc, 2007.

[10] P. Hui and J. Crowcroft, "How small labels create big improvements,” in Proc. IEEE ICMAN, March 2007.

[11] J. Burgess, B. Gallagher, D. Jensen, and B. N. Levine, "Max-prop: outing for vehicle-based disruption-tolerant networks," in INFOCOM 2006. 25th IEEE International Conference on Computer Communications. Proceedings, april 2006, pp. $1-11$.

[12] P. Hui, J. Crowcroft, and E. Yoneki, "Bubble rap: Social-based forwarding in delay-tolerant networks,"IEEE Transactions on Mobile Computing, vol. 10, pp. 1576-1589, 2011.

[13] E. Bulut, Z. Wang, and B. Szymanski, "Impact of social networks on delay tolerant routing," inGlobal Telecommunications Conference. GLOBECOM 2009. IEEE, 30 2009-dec. 4 2009, pp. 1-6, 2009. 